\title{
LIMITATION THEOREMS FOR SOME METHODS OF SUMMABILITY
}

\author{
Minoru Tanaka
}

\begin{abstract}
The object of this paper is to establish limitation theorems for the ordinary and also absolute generalized Nörlund methods which include some known results as special cases. We shall give a different proof of the recent result of $S$. Narang (Proc. Indian Acad. Sci. Sect. A 88 (1979), 115-123), and we get a generalization of the result of G. Das (J. London Math. Soc. 41 (1966), 685-692) which states the summability factors of the absolute Nörlund methods.
\end{abstract}

\section{Introduction}

The object of this paper is to establish limitation theorems for the $(N, p, \alpha)$ and $|N, p, \alpha|$ methods which include some known results as special cases. In Theorem 2 we shall give a different proof of a recent result of Narang ([6], Theorem 1 ). It is worth noting that in this theorem we cannot omit the condition $(i): \Delta(p * \alpha)_{n} \leq 0$, which was not mentioned in [6]. A counterexample is the case $(\mathrm{N}, p, \alpha)=(E, \lambda)$; in fact we may not apply the theorem to $(E, \lambda)$. Theorem 3 is a generalization of the result of Das ([1], Theorem 1) which states the summability factors of the absolute Nörlund methods.

Let $\left\{p_{\dot{n}}\right\}$ and $\left\{\alpha_{n}\right\}$ be given sequences of real numbers such that

$$
(p * \alpha)_{n}=\sum_{\nu=0}^{n} p_{n-v} \alpha_{v} \neq 0 \text { for all } n \geq 0 \text {, }
$$

Received 30 April 1980. 
and let $\sum a_{n}$ be a given infinite series with its partial sum $s_{n}$. If $t_{n} \rightarrow s$ as $n \rightarrow \infty$, where

$$
t_{n}=t_{n}^{p, \alpha}=\left(1 /(p * \alpha){ }_{n}\right) \sum_{\nu=0}^{n} p_{n-v^{\alpha} v^{s} v}
$$

then the series $\sum a_{n}$ is said to be summable (N, $\left.p, \alpha\right)$ to $s$ and we write $\sum a_{n}=s(N, p, \alpha)$ (see Das [2]). Also if the sequence $\left\{t_{n}^{p, \alpha}\right\}$ is of bounded variation

$$
\sum\left|t_{n}^{p, \alpha}-t_{n+1}^{p, \alpha}\right|<\infty
$$

the series $\sum a_{n}$ is said to be summable $|N, p, \alpha|$ and we write $\sum a_{n} \in|\mathrm{N}, p, \alpha|$. The method $(\mathrm{N}, p, \alpha)$ reduces to the Nörlund method $(N, p)$ when $\alpha_{n}=1$, to the method $(\bar{N}, \alpha)$ when $p_{n}=1$, and to the method $(E, \lambda)$ when $p_{n}=(\delta \lambda)^{n} / n !$ and $\alpha_{n}=\delta^{n} / n !(\lambda>0, \delta>0)$ (see Hardy [3], p. 179).

Throughout this paper we use the following notations. If $p_{0} \neq 0$, we define for $\left\{p_{n}\right\}$ a sequence $\left\{c_{n}\right\}$ such that

$$
(c * p)_{n}=\delta_{n, 0} \quad \text { (Kronecker delta). }
$$

We shall write $\left\{p_{n}\right\} \in M$ if $p_{n}>0, p_{n+1} / p_{n} \leq p_{n+2} / p_{n+1} \leq 1$ for all

$n \geq 0$. We denote $\Delta a_{n}=a_{n}-a_{n+1}, \nabla a_{n}=a_{n}-a_{n-1}$, $\Delta_{n} a_{n, \nu}=a_{n, \nu}-a_{n+1, \nu}$ and $a_{-1}=0$. A capital letter $K$ is an absolute constant, not necessarily the same at each occurrence.

\section{The main theorems}

Concerning the $(\mathbb{N}, p, \alpha)$ method, we have

THEOREM 1. Let $\left\{p_{n}\right\}$ and $\left\{\alpha_{n}\right\}$ be such that $\left\{p_{n}\right\} \in M$ and $\alpha_{n}>0$ for all $n$. Then $\sum a_{n}=s(N, p, \alpha)$ implies $s_{n}=s+o\left((p * \alpha)_{n} / \alpha_{n}\right)$ as 
$n \rightarrow \infty$

For the $|\mathrm{N}, p, \alpha|$ method, we have

THEOREM 2. Let $\left\{p_{n}\right\}$ and $\left\{\alpha_{n}\right\}$ be two positive sequences and suppose

(i) $\Delta(p * \alpha)_{n} \leq 0$ for all $n$,

(ii) $\sum\left|c_{n}\right|<\infty$,

(iii) $\left\{\alpha_{n} /(p * \alpha)_{n}\right\}$ is of bounded variation.

Then, for every series $\sum a_{n} \in|N, p, \alpha|$ with partial sum $s_{n}$, the sequence $\left\{s_{n} \alpha_{n} /(p * \alpha)_{n}\right\}$ is of bounded variation.

When $\alpha_{n}=1$ for all $n$, the conditions $(i)$ and (iii) are always satisfied, and we obtain a result of Kishore [4]. Also when $p_{n}=1$ for all $n$, the conditions $(i)$ and $(i i)$ hold and we get a result of Mohanty ([5], Lemma 3).

THEOREM 3. Let $\left\{p_{n}\right\}$ and $\left\{\alpha_{n}\right\}$ be such that

(i) $\sum\left|c_{n}\right|<\infty$,

(ii) $\sum_{\mu=0}^{n}\left|\nabla(p * \alpha)_{\mu}\right| \leq K\left|(p * \alpha)_{n}\right|$,

(iii) $\sum_{n=\nu+1}^{\infty}\left|1-\left(\alpha_{n} / \alpha_{n-1}\right)\right| \sum_{\mu=0}^{\nu}\left|c_{n-\mu}\right| \leq K$ for every $\nu \geq 0$.

Then a necessary and sufficient condition for $\sum \varepsilon_{n} a_{n}$ to be absolutely convergent whenever $\sum a_{n} \in|\mathrm{N}, p, \alpha|$ is

$$
\varepsilon_{n}=o\left(\alpha_{n} /(p * \alpha)_{n}\right)
$$

When $\alpha_{n}=1$ for all $n$, condition $(i i i)$ is always satisfied and we obtain a theorem of Das ([1], Theorem 1). On the other hand when $p_{n}=1$ for all $n$, condition $(i)$ is satisfied and ( $i i i)$ is equivalent to 
(iii)' $\alpha_{n} / \alpha_{n-1}=O(1)$,

so we have

COROLLARY. Let $\left\{\alpha_{n}\right\}$ be such that (iii)' holds and

$\sum_{\nu=0}^{n}\left|\alpha_{v}\right|=o\left((1 * \alpha)_{n}\right)$. Then a necessary and sufficient condition for

$\sum \varepsilon_{n} a_{n}$ to be absolutely convergent whenever $\sum a_{n} \in|\overline{\mathrm{N}}, \alpha|$ is $\varepsilon_{n}=O\left(\alpha_{n} /(1 * \alpha)_{n}\right)$.

\section{Proof of the theorems}

We need the following lemmas.

LEMMA 1 (Das [2]). Let $a_{n} \neq 0$ for alz $n$. If $\left\{t_{n}^{p}, \alpha\right\}$ is defined by (1.1), then

$$
s_{n}=\left(1 / \alpha_{n}\right) \sum_{\nu=0}^{n} c_{n-v}(p * \alpha) v_{v}^{t} v_{v}, \alpha \text { for } a z z n \text {. }
$$

LEMMA 2 (Kulza; see [3], Theorem 22). If $\left\{p_{n}\right\} \in M$, then $c_{0}>0, c_{n} \leq 0 \quad(n \geq 1)$ and $\sum_{n=0}^{\infty} c_{n} \geq 0$.

LEMMA 3 (see Peyerimhoff [7], Theorem II, 14). Let $A=\left(a_{n v}\right)$ be normal and regular, and let $\sigma_{n}=\sum_{\nu=0}^{n} a_{n \nu} s_{v}$. Suppose that $M_{K}(A)$ hold:

$$
\left|\sum_{\nu=0}^{m} a_{n \nu} s \nu\right| \leq K \cdot \sup _{\mu \leq m}\left|\sigma_{\mu}\right| \text { for } m \leq n \text {. }
$$

Then $\sum a_{n}=s(A)$ implies $s_{n}=s+o\left(1 / a_{n n}\right)$.

LEMMA 4 (Das [1], Lemma 2). If $y_{n}=\sum_{\nu=0}^{\infty} d_{n \nu^{x}}$ for azz $n$ where $\left\{d_{n v}\right\}$ is a double sequence, then a necessary and sufficient condition that the series $\sum\left|y_{n}\right|$ is convergent whenever $\sum\left|x_{n}\right|$ is convergent is that 


$$
\sum_{n=0}^{\infty}\left|d_{n v}\right| \leq K \text { for each } v \geq 0
$$

3.1. Proof of Theorem 1. By Lemma 3 it is sufficient to show that

$$
\left|\sum_{\nu=0}^{m}\left(p_{n-\nu^{\alpha} \nu^{\prime}} /(p * \alpha)_{n}\right) s_{\nu}\right| \leq \sup _{\mu \leq m}\left|t_{\mu}^{p, \alpha}\right| \text { for } m \leq n \text {. }
$$

Now by Lemma 2 we see $c_{0}>0, c_{n} \leq 0$ for $n \geq 1$. So we have

$$
\begin{aligned}
\sum_{\nu=\mu}^{m} p_{n-\nu^{c} \nu-\mu} & =\sum_{\nu=0}^{m-\mu} p_{n-\nu-\mu} c_{\nu} \\
& =\sum_{\nu=0}^{n-\mu} p_{n-\nu-\mu^{c} \nu}-\sum_{\nu=m-\mu+1}^{n-\mu} p_{n-\nu-\mu^{c} \nu} \\
& =\delta_{n-\mu, 0}-\sum_{\nu=m-\mu+1}^{n-\mu} p_{n-\nu-\mu^{c} \nu} \\
& \geq 0,
\end{aligned}
$$

since $m-\mu+1 \geq 1$. Hence we get

$$
\begin{aligned}
\sum_{\mu=0}^{m} \mid \sum_{\nu=\mu}^{m}\left(p_{n-\nu^{\alpha} \nu^{\prime}}(p * \alpha)_{n}\right)\left(c_{\nu-\mu} / \alpha_{\nu}\right) & (p * \alpha)_{\mu} \mid \\
& =\sum_{\mu=0}^{m}\left((p * \alpha)_{\mu} /(p * \alpha)_{n}\right) \sum_{\nu=\mu}^{m} p_{n-\nu^{c} \nu-\mu} \\
& =\left(1 /(p * \alpha)_{n}\right) \sum_{\nu=0}^{m} p_{n-\nu} \sum_{\mu=0}^{\nu}(p * \alpha)_{\mu}{ }^{c} v-\mu \\
& =\left(1 /(p * \alpha)_{n}\right) \sum_{\nu=0}^{m} p_{n-\nu}{ }^{\alpha} v \\
& \leq 1 \text { for } m \leq n .
\end{aligned}
$$

But this result is a necessary and sufficient condition for $M_{1}((N, p, \alpha))$ since by Lemma 1 the inverse matrix of $(N, p, \alpha)$ is $\left(\alpha_{n v}^{\prime}\right)$ where $a_{n v}^{\prime}=c_{n-v}(p * \alpha)_{v} / \alpha_{n}(n \geq v),=0(n<\gamma) \quad($ see Peyerimhoff [7], p. 31).

Therefore we have the conclusion.

3.2. Proof of Theorem 2. By Abel's transformation it follows from Lemma 1 that 
378

Minor Tanka

$$
\begin{aligned}
s_{n} \alpha_{n} & =\sum_{\nu=0}^{n-1}\left(\Delta t_{\nu}\right) \sum_{\mu=0}^{\nu} c_{n-\mu}(p * \alpha)_{\mu}+t_{n} \sum_{\mu=0}^{n} c_{n-\mu}(p * \alpha)_{\mu} \\
& =\sum_{\nu=0}^{n-1}\left(\Delta t_{\nu}\right) \sum_{\mu=0}^{\nu} c_{n-\mu}(p * \alpha)_{\mu}+t_{n}{ }_{n},
\end{aligned}
$$

and also

$$
\begin{aligned}
\Delta\left(s_{n} \alpha_{n}\right) & =\sum_{\nu=0}^{n+1}\left(c_{n-\nu}-c_{n+1-\nu}\right)(p * \alpha)_{\nu} t_{\nu} \\
& =\sum_{\nu=0}^{n}\left(\Delta t_{\nu}\right)\left\{c_{n-\nu}(p * \alpha)_{\nu+1}-\sum_{\mu=0}^{\nu+1} c_{n+1-\mu} \nabla(p * \alpha)_{\mu}\right\}+t_{n+1}\left(\alpha_{n}-\alpha_{n+1}\right) .
\end{aligned}
$$

Hence we have

$$
\begin{aligned}
& \Delta\left(s_{n}{ }_{n} /(p * \alpha)_{n}\right) \\
& =\left(\Delta\left(1 /(p * \alpha)_{n}\right)\right) s_{n} \alpha_{n}+\left(1 /(p * \alpha)_{n+1}\right) \Delta\left(s_{n} \alpha_{n}\right) \\
& =\left(\Delta\left(1 /(p * \alpha){ }_{n}\right)\right)\left\{\sum_{\nu=0}^{n-1}\left(\Delta t_{\nu}\right) \sum_{\mu=0}^{\nu} c_{n-\mu}(p * \alpha)_{\mu}+t_{n}{ }^{\alpha} n\right\}+\left(1 /(p * \alpha)_{n+1}\right) \\
& \times\left\{\sum_{\nu=0}^{n}\left(\Delta t_{\nu}\right) c_{n-\nu}(p * \alpha)_{\nu+1}-\sum_{\nu=0}^{n}\left(\Delta t_{\nu}\right) \sum_{\mu=0}^{\nu} c_{n+1-\mu} \nabla(p * \alpha)_{\mu}+t_{n+1}\left(\alpha_{n}-\alpha_{n+1}\right)\right\} \\
& =\left(\Delta\left(1 /(p * \alpha)_{n}\right)\right) \sum_{\nu=0}^{n-1}\left(\Delta t_{\nu}\right) \sum_{\mu=0}^{\nu} c_{n-\mu}(p * \alpha)_{\mu} \\
& +\left(1 /(p * \alpha)_{n+1}\right) \sum_{v=0}^{n}\left(\Delta t_{v}\right) c_{n-v}(p * \alpha)_{v+1} \\
& -\left(1 /(p * \alpha)_{n+1}\right) \sum_{\nu=0}^{n}\left(\Delta t_{\nu}\right) \sum_{\mu=0}^{\nu} c_{n+1-\mu} \nabla(p * \alpha)_{\mu} \\
& +\Delta\left(\alpha_{n} t_{n} /(p * \alpha)_{n}\right)-\left(\alpha_{n} /(p * \alpha)_{n+1}\right)\left(\Delta t_{n}\right) \text {. }
\end{aligned}
$$

Therefore we get

https://doi.org/10.1017/S0004972700006699 Published online by Cambridge University Press 


$$
\begin{aligned}
& \sum_{n=0}^{\infty}\left|\Delta\left(s_{n} \alpha_{n} /(p * \alpha){ }_{n}\right)\right| \\
& \leq \sum_{n=0}^{\infty}\left|\left(\Delta\left(1 /(p * \alpha)_{n}\right)\right) \sum_{\nu=0}^{n-1}\left(\Delta t_{\nu}\right) \sum_{\mu=0}^{\nu} c_{n-\mu}(p * \alpha)_{\mu}\right| \\
& +\sum_{n=0}^{\infty}\left|\left(1 /(p * \alpha)_{n+1}\right) \sum_{\nu=0}^{n}\left(\Delta t_{v}\right) c_{n-\nu}(p * \alpha)_{v+1}\right| \\
& +\sum_{n=0}^{\infty}\left|\left(1 /(p * \alpha)_{n+1}\right) \sum_{\nu=0}^{n}\left(\Delta t_{\nu}\right) \sum_{\mu=0}^{\nu} c_{n+1-\mu} \nabla(p * \alpha)_{\mu}\right| \\
& +\sum_{n=0}^{\infty}\left|\Delta\left(\alpha_{n} t_{n} /(p * \alpha)_{n}\right)\right|+\sum_{n=0}^{\infty}\left|\left(\alpha_{n} /(p * \alpha)_{n+1}\right)\left(\Delta t_{n}\right)\right| \\
& =J_{1}+J_{2}+J_{3}+J_{4}+J_{5} \text {, say. }
\end{aligned}
$$

Then by (i) and (ii),

$$
\begin{aligned}
& J_{1} \leq \sum_{n=0}^{\infty}\left(\Delta\left(1 /(p * \alpha)_{n}\right)\right) \sum_{\nu=0}^{n-1}\left|\Delta t_{\nu}\right| \sum_{\mu=0}^{\nu}\left|c_{n-\mu}\right|(p * \alpha)_{\mu} \\
& =\sum_{\nu=0}^{\infty}\left|\Delta t_{\nu}\right| \sum_{n=\nu+1}^{\infty}\left(\Delta\left(1 /(p * \alpha)_{n}\right)\right) \sum_{\mu=0}^{\nu}\left|c_{n-\mu}\right|(p * \alpha)_{\mu} \\
& \leq \sum_{\nu=0}^{\infty}\left|\Delta t_{\nu}\right|(p * \alpha)_{\nu}\left(\sum_{\mu=0}^{\infty}\left|c_{\mu}\right|\right) \sum_{n=v+1}^{\infty} \Delta\left(1 /(p * \alpha)_{n}\right) \\
& \leq K \sum_{\nu=0}^{\infty}\left|\Delta t v_{\nu}\right|<\infty \text {, } \\
& \left.J_{2} \leq \sum_{n=0}^{\infty}\left(1 /(p * \alpha)_{n+1}\right) \sum_{\nu=0}^{n}\left|\Delta t_{v}\right|\left|c_{n-v}\right| ! p * \alpha\right)_{\nu+1} \\
& =\sum_{\nu=0}^{\infty}\left|\Delta t_{\nu}\right| \sum_{n=\nu}^{\infty}\left(1 /(p * \alpha)_{n+1}\right)\left|c_{n-\nu}\right|(p * \alpha)_{\nu+1} \\
& \leq \sum_{\nu=0}^{\infty}\left|\Delta t_{\nu}\right| \sum_{n=0}^{\infty}\left|c_{n}\right|<\infty,
\end{aligned}
$$




$$
\begin{aligned}
J_{3} & \leq \sum_{n=0}^{\infty}\left(1 /(p * \alpha)_{n+1}\right) \sum_{\nu=0}^{n}\left|\Delta t_{\nu}\right| \sum_{\mu=0}^{\nu}\left|c_{n+1-\mu}\right| \nabla(p * \alpha)_{\mu} \\
& =\sum_{\nu=0}^{\infty}\left|\Delta t_{\nu}\right| \sum_{n=\nu}^{\infty}\left(1 /(p * \alpha)_{n+1}\right) \sum_{\mu=0}^{\nu}\left|c_{n+1-\mu}\right| \nabla(p * \alpha)_{\mu} \\
& \leq K \cdot \sum_{\nu=0}^{\infty}\left|\Delta t_{\nu}\right|\left(1 /(p * \alpha)_{\nu+1}\right) \sum_{\mu=0}^{\nu} \nabla(p * \alpha)_{\mu} \\
& \leq K \cdot \sum_{\nu=0}^{\infty}\left|\Delta t_{\nu}\right|<\infty .
\end{aligned}
$$

Also we have by ( $i$ ii), and by our assumption,

$$
\begin{aligned}
& J_{4}=\sum_{n=0}^{\infty}\left|\Delta\left(\alpha_{n} t_{n} /(p * \alpha)_{n}\right)\right|<\infty, \\
& J_{5} \leq K \cdot \sum_{n=0}^{\infty}\left|\Delta t_{n}\right|<\infty .
\end{aligned}
$$

Therefore it follows that

$$
\sum_{n=0}^{\infty}\left|\Delta\left(S_{n} \alpha_{n} /(p * \alpha)_{n}\right)\right|<\infty .
$$

Thus the proof of our theorem is completed.

3.3. Proof of Theorem 3. Sufficiency. By Lemma 1 and by Abel's transformation we have, for $n \geq 1$,

(3.1) $a_{n}=\sum_{\nu=0}^{n-1} \Delta t \sum_{\mu=0}^{\nu}\left(\Delta_{n}\left(c_{n-\mu} / \alpha_{n}\right)\right)(p * \alpha)_{\mu}$

$$
+t_{n} \sum_{\mu=0}^{n}\left(\nabla_{n}\left(c_{n-\mu} / \alpha_{n}\right)\right)(p * \alpha)_{\mu}
$$

$$
=\sum_{\nu=0}^{n-1} \Delta t_{\nu} \sum_{\mu=0}^{\nu}\left(\nabla_{n}\left(c_{n-\mu} / \alpha_{n}\right)\right)(p * \alpha)_{\mu},
$$

since (1.2) implies

$$
\begin{aligned}
\sum_{\mu=0}^{n}\left(\nabla_{n}\left(c_{n-\mu} / \alpha_{n}\right)\right)(p * \alpha)_{\mu} & =\left(1 / \alpha_{n}\right)(c * p * \alpha)_{n}-\left(1 / \alpha_{n-1}\right)(c * p * \alpha)_{n-1} \\
& =0 .
\end{aligned}
$$


Moreover using Abel's transformation again,

$$
\begin{aligned}
\sum_{\mu=0}^{\nu}\left(\nabla_{n}\left(c_{n-\mu} / \alpha_{n}\right)\right)(p & * \alpha)_{\mu}=\left(\nabla\left(1 / \alpha_{n}\right)\right) \sum_{\mu=0}^{\nu} c_{n-\mu}(p * \alpha)_{\mu} \\
& +\left(1 / \alpha_{n-1}\right) \sum_{\mu=0}^{\nu} c_{n-\mu} \nabla(p * \alpha)_{\mu}-\left(1 / \alpha_{n-1}\right) c_{n-1-\nu}(p * \alpha)_{\nu}
\end{aligned}
$$

and it follows that

$$
\begin{aligned}
\sum_{n=1}^{\infty}\left|\varepsilon_{n} \alpha_{n}\right| & =\sum_{n=1}^{\infty}\left|\varepsilon_{n} \sum_{\nu=0}^{n-1} \Delta t_{v}\{\}\right| \\
\leq & \sum_{n=1}^{\infty}\left|\varepsilon_{n} \sum_{v=0}^{n-1} \Delta t_{v}\left(\nabla\left(1 / \alpha_{n}\right)\right) \sum_{\mu=0}^{\nu} c_{n-\mu}(p * \alpha)_{\mu}\right| \\
& +\sum_{n=1}^{\infty}\left|\varepsilon_{n} \sum_{v=0}^{n-1} \Delta t_{v}\left(1 / \alpha_{n-1}\right) \sum_{\mu=0}^{\nu} c_{n-\mu} \nabla(p * \alpha)_{\mu}\right| \\
& +\sum_{n=1}^{\infty}\left|\varepsilon_{n} \sum_{v=0}^{n-1} \Delta t_{v}\left(1 / \alpha_{n-1}\right) c_{n-1-\nu}(p * \alpha)\right| \\
= & \Sigma_{1}+\Sigma_{2}+\Sigma_{3}, \text { say. }
\end{aligned}
$$

Now, by (2.1) with (ii), we get

$$
\begin{aligned}
\left|\varepsilon_{n}\right| & \leq K\left|\alpha_{n}\right|\left(\left|(p * \alpha)_{n}\right|\right)^{-1} \leq K\left|\alpha_{n}\right|\left(\sum_{\mu=0}^{n}\left|\nabla(p * \alpha)_{\mu}\right|\right)^{-1} \\
& \leq K\left|\alpha_{n}\right|\left(\sum_{\mu=0}^{\nu}\left|\nabla(p * \alpha)_{\mu}\right|\right)^{-1} \text { for } n \geq \nu .
\end{aligned}
$$

Hence we have by $(i i i)$, 
382

Minor Tanka

$$
\begin{aligned}
\Sigma_{1} & \leq \sum_{n=1}^{\infty}\left|\varepsilon_{n}\right| \sum_{\nu=0}^{n-1}\left|\Delta t_{\nu}\right|\left|\nabla\left(1 / \alpha_{n}\right)\right| \sum_{\mu=0}^{\nu}\left|c_{n-\mu}\right|\left|(p * \alpha)_{\mu}\right| \\
& =\sum_{\nu=0}^{\infty}\left|\Delta t_{\nu}\right| \sum_{n=v+1}^{\infty}\left|\varepsilon_{n}\right|\left|\nabla\left(1 / \alpha_{n}\right)\right| \sum_{\mu=0}^{\nu}\left|c_{n-\mu}\right|\left|(p * \alpha)_{\mu}\right| \\
& \left.\leq \sum_{\nu=0}^{\infty}\left|\Delta t_{\nu}\right| \sum_{n=v+1}^{\infty}\left|\varepsilon_{n}\right|\left|\nabla\left(1 / \alpha_{n}\right)\right| \sum_{i=0}^{\nu}\left|\nabla(p * \alpha)_{i}\right|\right) \sum_{\mu=0}^{n}\left|c_{n-\mu}\right| \\
& \leq K \sum_{\nu=0}^{\infty}\left|\Delta t_{\nu}\right| \sum_{n=v+1}^{\infty}\left|1-\left(\alpha_{n} / \alpha_{n-1}\right)\right| \sum_{\mu=0}^{\nu}\left|c_{n-\mu}\right| \\
& \leq K \cdot \sum_{\nu=0}^{\infty}\left|\Delta t_{\nu}\right|<\infty .
\end{aligned}
$$

Also by (i) and since (iii) implies $\alpha_{n} / \alpha_{n-1}=O(1)$, we get

$$
\begin{aligned}
\Sigma_{2} & \leq \sum_{n=1}^{\infty}\left|\varepsilon_{n}\right|\left|1 / \alpha_{n-1}\right| \sum_{\nu=0}^{n-1}\left|\Delta t_{\nu}\right| \sum_{\mu=0}^{\nu}\left|c_{n-\mu}\right|\left|\nabla(p * \alpha)_{\mu}\right| \\
& =\sum_{\nu=0}^{\infty}\left|\Delta t_{\nu}\right| \sum_{n=\nu+1}^{\infty}\left|\varepsilon_{n}\right|\left|1 / \alpha_{n-1}\right| \sum_{\mu=0}^{\nu}\left|c_{n-\mu}\right|\left|\nabla(p * \alpha)_{\mu}\right| \\
& \leq K \sum_{\nu=0}^{\infty}\left|\Delta t_{\nu}\right| \sum_{\mu=0}^{\nu}\left|\nabla(p * \alpha)_{\mu}\right|\left|\sum_{\mu=0}^{\nu}\right| \nabla(p * \alpha)_{\mu}||^{-1} \sum_{n=\nu+1}^{\infty}\left|\alpha_{n} / \alpha_{n-1}\right|\left|c_{n-\mu}\right| \\
& \leq K \sum_{\nu=0}^{\infty}\left|\Delta t_{\nu}\right| \sum_{n=\nu+1}^{\infty}\left|c_{n-\nu-1}\right| \\
& \leq K \sum_{\nu=0}^{\infty}\left|\Delta t_{\nu}\right|<\infty .
\end{aligned}
$$

Similarly we have

$$
\begin{aligned}
\Sigma_{3} & \leq \sum_{n=1}^{\infty}\left|\varepsilon_{n}\right| \sum_{\nu=0}^{n-1}\left|\Delta t_{\nu}\right|\left|1 / \alpha_{n-1}\right|\left|c_{n-1-\mu}\right|\left|(p * \alpha)_{\nu}\right| \\
& =\sum_{\nu=0}^{\infty}\left|\Delta t_{\nu}\right|\left|(p * \alpha)_{\nu}\right| \sum_{n=v+1}^{\infty}\left|\varepsilon_{n}\right|\left|1 / \alpha_{n-1}\right|\left|c_{n-1-\nu}\right| \\
& \leq K \sum_{\nu=0}^{\infty}\left|\Delta t_{\nu}\right|\left|(p * \alpha)_{\nu}\right|\left(\sum_{\mu=0}^{\nu}\left|\nabla(p * \alpha)_{\mu}\right|\right)^{-1} \sum_{n=v+1}^{\infty}\left|\alpha_{n} / \alpha_{n-1}\right|\left|c_{n-1-\nu}\right| \\
& \leq K \sum_{\nu=0}^{\infty}\left|\Delta t_{\nu}\right|<\infty .
\end{aligned}
$$

https://doi.org/10.1017/S0004972700006699 Published online by Cambridge University Press 
Hence it follows that $\sum_{n=0}^{\infty}\left|\varepsilon_{n} a_{n}\right|<\infty$, and the proof of the sufficiency part is completed.

Necessity. From (3.1) we have, for $n \geq 1$,

$$
\varepsilon_{n} a_{n}=\sum_{\nu=0}^{n} \Delta t v_{n, \nu}
$$

where

$$
d_{n, \nu}= \begin{cases}\varepsilon_{n} \sum_{\mu=0}^{\nu}\left(\nabla_{n}\left(c_{n-\mu} / \alpha_{n}\right)\right)(p * \alpha)_{\mu} & (\nu \leq n), \\ 0 & (\nu>n) .\end{cases}
$$

Now, by Lemma 4, a necessary condition for $\sum\left|\varepsilon_{n} a_{n}\right|$ to be convergent whenever $\sum a_{n}$ is summable $|\mathrm{N}, p, \alpha|$ is that $\sum_{n=v+1}^{\infty}\left|d_{n, v}\right| \leq K$. Hence it is necessary that $d_{v+1, v}=O(1)$ as $v \rightarrow \infty$. But

$$
\begin{aligned}
d_{v+1, \nu} & =\varepsilon_{v+1} \sum_{\mu=0}^{\nu}\left(\nabla_{v}\left(c_{v+1-\mu} / \alpha_{v+1}\right)\right)(p * \alpha)_{\mu} \\
& =-\varepsilon_{v+1}\left(c_{0} / \alpha_{v+1}\right)(p * \alpha)_{\nu+1} .
\end{aligned}
$$

Therefore the condition (2.1) is necessary.

This completes the proof of Theorem 3 .

\section{References}

[1] G. Das, "On the absolute Förlund summability factors of infinite series", J. London Math. Soc. 41 (1966), 685-692.

[2] G. Das, "On some methods of summability", Quart. J. Math. Oxford Ser. 17 (1966), 244-256.

[3] G.H. Hardy, Divergent series (Clarendon Press, Oxford, 1949).

[4] Nand Kishore, "A limitation theorem for absolute Ijörlund summability", J. London Math. Soc. (2) 4 (1971/2), $240-244$. 
[5] R. Mohanty, "On the absolute Riesz summability of Fourier series and allied series", Proc. London Math. Soc. (2) 52 (1951), 295-320.

[6] S. Narang, "Limitation theorems for absolute Nörlund summability", Proc. Indian Acad. Sci. Sect. A 88 (1979), 115-123.

[7] A. Peyerimhoff, Lectures on sumability (Lecture Notes in Mathematics, 107. Springer-Verlag, Berlin, Heidelberg, New York, 1969).

Department of Mathematics, Faculty of Science,

Chiba University,

Chiba 280 , Japan. 